\title{
Granuloma eosinófilo e Histiocitosis
}

\author{
Ins. Tomik I'rtegs*, Francisco Pararla**, \\ Jajs Fucutes*" Davisl Mirkin***
}

En esta prescntación analizaremos los pacientes alfectiklos de Granuloma eosinófilo tel sistema esquelético, controladiss en nuestro Servirio durante less últimos años. Como estid enticlud mórbida es parte integrance de la Histicitosis, haremos mu breve descripeión de lat misma.

La Histiccitosis comprende un grupo de enfermedades cuya alteración primordial es I: proliferación de las células lisiociticas en los tejidlos, ya sea de disposición focal o diseminacli. Son alecciones lifíciles de clasificar por sus semejanzas clinicas y anatomopatológicas, además de falta de limites precisos entre ellas. ya que pucalen exolucionar de umil forma a otra. Sin embarqo, hay algumas cararterísticas que ayulan a diferenciarlas: edach de comicno, localizada o difusiz, lenta o rápida erolución, sitio anatómico de la afección. herencia y respucsta al tratamicnto.

Una clasificación que nos parece más didáctica y que al mismo tiempo nus familiariar con la sincnimia de estas enfermalades, es la que aparece en un reciente trabajo cle Leiken (Tabli 1).

I als mis conocidas de éblas on las que J,ichtenstein denomino Histiocitosis $\mathrm{X}$ delsido a su ejologít y patogenia no precisalas. Combprenden, las formas diseminatlas, Lint. de Leiterer-Siwe (agurla, maligna) y Enf, de HandRio.

- Servicio de Cirugía Infantil. Iospieal Ruberto del

- Servicio ele Anatomía Patológicat. Hospital Rober. (0) 1 cl Rio.

Rev. Chilena de Pedituria, vol. 47, N? I, 1976
Sichuller-Ciluistian (crónicn, Irenigna), y und forma colitaria, el ciranuloma cosimófilo.

lat eciologín es desconocida y la patogenia podrti explicarse como una respuesta a un cxtímulo del exterior con un paciente $\alpha m$ umal reacividad especial $y$ cuyas lesiones se detienen en su alesarrollo cercano a lat malignielad absolutá.

\section{'fabla 1}

CAMSIFICACION INE IA HASTOCITOSAS

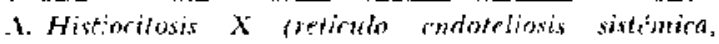
retirulo andoteliosis histiocition).

1. Diseminata

i) Agucla (maligua, lecterer-Siwr)

b) Crónica (benigna-kite. Hantl-sehuller-Chris. (iais).

2. Fomma solitaria (gramuloma cosionfito) .

13. Hixtiocitosis fomiliar

1. Retisalo endoteliosis henolagocítica [amilias (linfohistiocitosis familiar. Infohistiocilosis cri. móitgocltica familiar. reticulosis bistiocitica $f_{\mathrm{a}}$. miliary.

2. Histiocionsis asociatla com cosinofilia y deficicucia prin!ariat inm!nuolingica.

3. Reticulo encleteliosix hijxerglobulinćmica.

C. Histiociade's maligna

\section{MATERIAL Y METODO}

Nuestro estudio comprende: 9 pacientes, en el Servicio de Cirugía Infantil y Oriopelia, del 
Hospital Roberto del Río, entre los años 1965 y 1974.

Dos de elios corresponden a la forma diseminada y siete a la forma solitaria o granuloma eosinófilo propiamente tal (Tabla 2).

Tabla 2

\section{HISTIOCTROSIS $\mathrm{x}$. CASUISTICA}

\begin{tabular}{ll}
\hline Diagnossico & No pac. \\
\hline Fornas diseminadas & \\
Enf, de Letterer-Siwe & 1 \\
Enf. de Hand-Schuller-Chrìstian & 1 \\
Forma solitaria & \\
Granulonna cokinófilo & 7 \\
\hline Total & 0
\end{tabular}

Seis de los nueve casos se encuentran en niños eritre los 4 y 7 años cle edad; el menor de la serie corresponde a uno de 2 años afectado de la forma diseminada maligna (Figura NQ l).

\section{Figura $N^{\mathrm{c}} 1$}

MISTIOCITOSIS $X$

UNCIDENGIA POR EQAD

$N^{O}$ DE CASOS

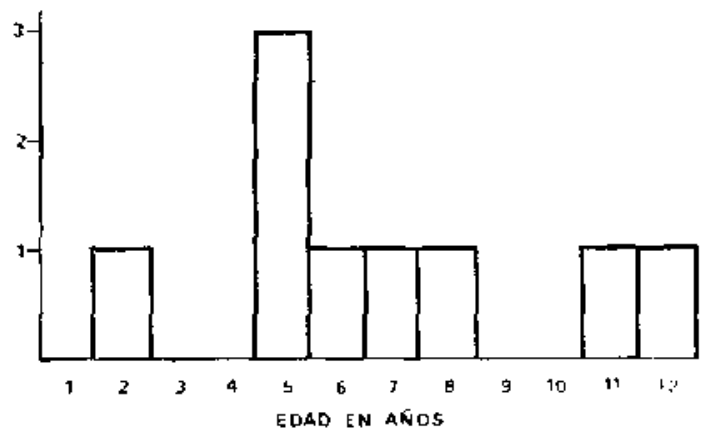

Con respecto al scxo, fue más frecuente en las niñas que en los varones, no concordando ello con otros attores.

\section{Sintomatologia}

En lo que se refiere al cuadro clínico, en la Corma solitaria o granuloma eosinófilo propiamente dicho, los sintomas más importantes fueron dolor osteoarticular y tumefacción concomitante de las partes blandas, acompañados cle signos vagos de compromiso del estado general (Tabla 3).
Tabla 3

GRANULOMA EOSINOFILO. SINTOMATOLOGLA

\begin{tabular}{lcr}
\hline Sintomas y signos & No pac. & $\%$ \\
\hline & & \\
Dolor osteoarticular & 7 & $100 \%$ \\
Tunefaccion de partes blandas & 3 & $42 \%$ \\
Fiebre & 2 & $28 \%$ \\
Palidez & 2 & $28 \%$ \\
Anorexia & 1 & $14 \%$ \\
Signos PIcuropulmonares & 1 & $14 \%$ \\
\hline
\end{tabular}

Nuestros dos pacientes con formas diseminatlas presentaron también los síntomas dependientes de las lesiones óseas, es decir dolor osteoarticular y tumefacción de las partes blandas correspondientes. Ademais, ambos pa. decieron de exoftalmia unilateral por inva. sión orbitaria y retrorbitaria de la lesión gra. nulomatosa. Otros síntomas a considerar fue ron la otitis media, de tipo proliferativo, los pleuropuimonares $y$ las alteraciones del estaklo general.

Las lesiones óseas fueron únicas en $\operatorname{los} 7$ pacientes de Granuloma eosinófilo, siendo múltiples en los otros 2 pacientes. Se localizaron mis frecuentemente en la pelvis, en el hueso ilíaco derecho en los 5 niños, siguiendo en orden de frecuencia las lesiones crantanas, (sienclo esta localización Ja más frecuente para diversos autores); en el tórax, 2 presentaron lesiones costales y 1 del omóplato; por último 1 tenia lesiones en el térnur (Tabla 4).

Tabla 4

HISTrOCITOSIS $x$. LESIONES OSEAS

\begin{tabular}{lcc}
\hline Localización & NP pac. & $\%$ \\
\hline Pelvis & 5 & $55 \%$ \\
Cráneo & 4 & $45 \%$ \\
Tórax & 5 & $\$ 3 \%$ \\
Extremidades Inferiores & 1 & $11 \%$ \\
\hline
\end{tabular}

El estudio radiológico éectuado mostró en la mayoría de los casos la imagen típica de la lesión ósea, redondeada, transparente, de lí. mites densos y precisos, correspondiendo a proceso osteoliticu con reacción osteoesclerótica a su alrededor. 
Para el djagnnóstico de la histiocitosis es imprescindible el estudio histopatológico correspondiente. El término "Granuloma eosinófilo" es admirablemente descriptivo para la mayoria de cstas lesiones, cuando se las examina microscópicamente. La matriz consiste usualmente de una masá reticulada de histiocitos infiltrada por leucocitos eosinófilos. Estos últimos pucden ser tan abundantes como para fonnar masas sóliclas que ocupan una grat parte del tejido tincondo el aret de un rojo brillante (Figura No 2).

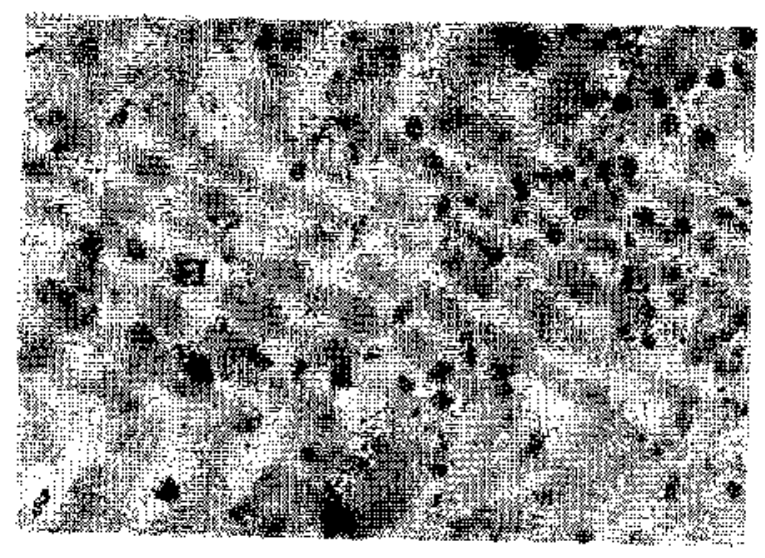

Figura 2 Microlotografia. Jroliferación de cilulas hisliocitarias (H) y acíiurulo de polinucleares cosimófilos (E). I a flecha sệ̃aIa una cólula gigante reultinucleacia. Hematoxilina-cosina. Ob $40 \mathrm{x}$.

Fin ocasiones las rélulas cosinófilas, ya sea formas macluras o más jóvenes (mielocitos eosinófilos) pueden ser escasas o aun faltar. Puede haber prevalencia de linforitos, plasmocitos o leucocitos neutrófilos. En este caso puede plantearse el diagnóstico de osteomielitis (Figura No 3). Ocasionalmente la lesión puede consistir en su mayor parte de hisioci. tos bastante inmaduros. Este tjpo de lesiones ha Ilevado en el pasado a diagnósticos erróneos de sarcoma reticular o mieloma. Con la "ma. duración" del proceso, material lipoídeo pue: de acumularse en el citoplasma de los histio. citos que adquieren aspecto xantomatoso (Fi. gura No 4).

La necrosis estimula la fagocitosis y se forman células gigantes multinucleadats, proba.

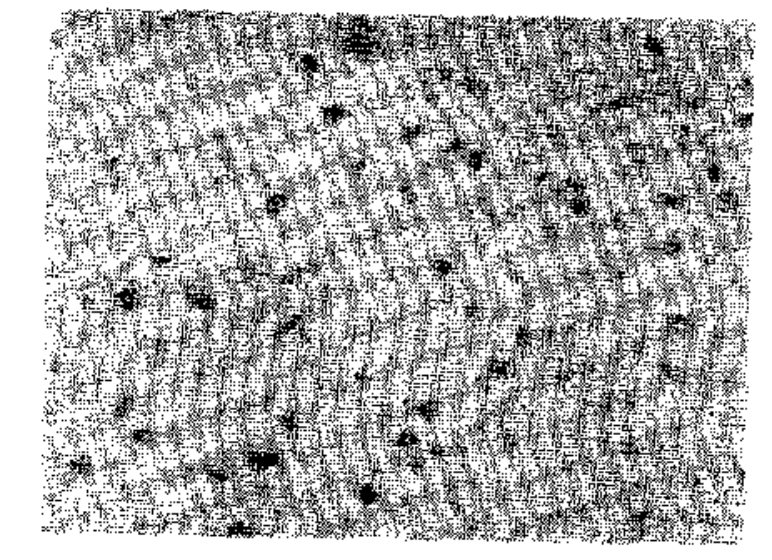

Jigura 4 Microfotografia, Acúnulo de histiocitos con citoplasmas cargados de lipoide. Hematoxilina-eosina. Ob. $40 \mathrm{x}$.

blemente por aglomeración de histiocitos (Figura $x^{\top}$ 5). El lipoide puede acumularse y formar cristales que aparezcan como hendiduras o espiculas negativas en las preparaciones hátjiuales. Gradualmente el exudado inflamatorio disminciye $y$ los fibroblastos co mienzan a dominar el cuadro hasta que la lesión cura por fibrosis total.

En la serie examinada 7 de los casos presentaban el aspecto descrito como clásico; eti algunos de los cortes 2 de ellos se acompañaban de abundantes células gigantes (formas (liseminadas). En un caso se encontraban islotes puros xantomatosos en medio de es. troma Libroso y en otro caso correspondiente " lesión de costilla tenía características "os- 


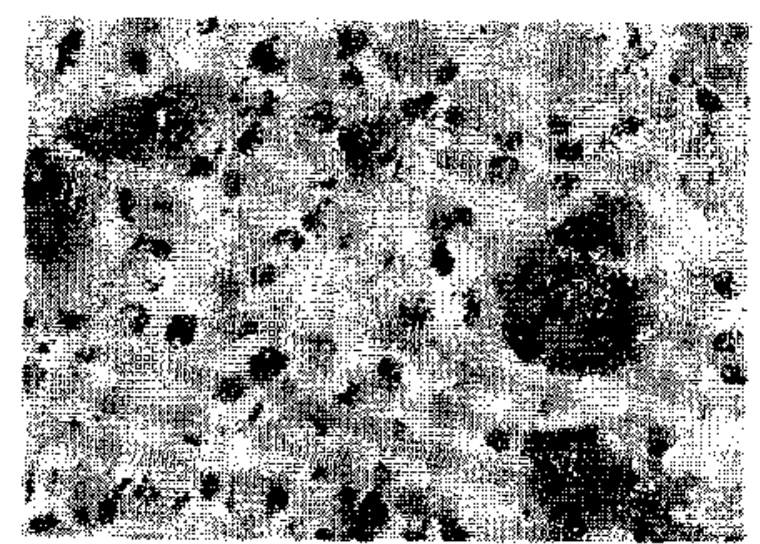

Figura 5 Microfolografle. C'élnlas gigantes multinu. cledas. En la mayoría de sus núcteos se reconocen las características de elcuncmos histionitarios. Hemiatoxilina-cosing. Ob $40 \mathrm{x}$.

tcomiclí.icas". El pronóstico es bueno cuando la histiocitosis afucta a un solo hueso romo en 7 de los pacientes ale esta serie (labla 5$)$.

\section{Tabla 5}

\section{]RONOOSTICO}

(1.ucdya, Olscrman-Fker. Campbill)

Grupo A: Cuando la enferuuctad afecta it in hueso. (a)upo B: Cuanclo afecta a mis de un hucso

Goupo c: l'́n huesu y tejiclos blandos

(imupo) I); Sólo tejitos lylazitos.

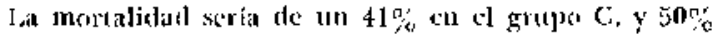
(n) (I 1).

\section{Tratamisto}

El tratamiento sairirgico efectuado consistio en la extirpación alel tumor que era blindo, friable, no encipsulato, de color anarillo-verdoso, no hemorrágico. Se encombabil acupando la oquedacla úsca producida, de bordes escleróticos e irregulares, a Ios que se le realisó rureaje, palra extracicion total de probables restos granulomatosos. En dos casos con (aviclades óseats gancles, se rellenaron Ias mismas con tejicló óseo coponjoso del mismo jariente.

Se realizci extirpación total en loss 7 njños afcotados de granuloma eosinólilo solitatio. En 5 de ellos se comprobó aración clínica y radjológica de la lesión en contmoles postrio. res al año. No se controlaron 2 niños. En las formá cliseminatlas se efectuó extirpación parcial de las Jesiones; más quimioterapia en la atguda maligna, encontrindose bicn al año de control, y radtoterajia en la croblica benigna, en buenas condiciones a los 3 años de control.

\section{RESLMEN}

Se anlizan niồos atectatos de Granuloma Eesimbililo osen que constataron an el Servicio rle (imuga del Hospital Roberto del Rio durante el petiolo de 1965 a 1974 . Se describen lit sintomatologia, diagnóstico lristologi(a) y el tritumicnto guirúrgins clectuids.

\section{SUMMTRY}

9) Children arfected of Losinophilir: granutoma of Jone werc seen in the Robzr.o del Rio Hospital Seqvice of Surgery aluring the period from 1965 to 1974. Symptomatology, histologiv: dirgnosis and surgical treatment performed are clescribed.

\section{REIFRENCIAS}

1. Argerter. E., Kilkpatrick, S. A., Ortheperdic Discases. W. B. Sautulers. Phi]adelphia: ] 968.

2. Hauzḱ. J. et al.. Cintmuknun cosinófilo (thistiocitosis

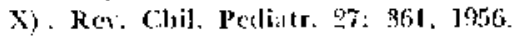

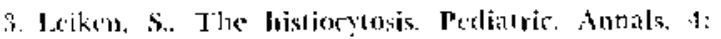
$45,195 \%$

1. Nelsoti. W. E. et al. Trataciu de Pecliatria. fo ed. Bacelona. Salvat, 197s. V. 2.

7. Orcman, It. A., Idiopathic listiocylosis. Peclintric: 28: 307.1961 .

6. Ocommer, J. 1\%. Radio'ogy and Pediatric. New relationships. Pedias'. Clin, $X$ Ancr gl: 329. lint

7. Sallas. M., Ials Neoplasias en los nif́os. la Ell. Ealicioncs Médicas de:l flompital Infantil de Méxics, 1968. 